\title{
Nuclear Broadening Effects on Hard Prompt Photons at CERN-SPS and BNL-RHIC Energies
}

\section{Gerland ${ }^{* \dagger}$ and Horst Stöcker}

Institut für Theoretische Physik der J.W. Goethe-Universität, Robert-Mayer-Str. 8-10, D-60054 Frankfurt a.M., Germany E-mail: 'gerland@th.physik.uni-frankfurt.de',

\section{A. Dumitru}

Physics Department, Columbia University, 538 West 120th Street, New York, NY10027, $U S A$

\section{Frankfurt}

Tel Aviv University, Tel Aviv, Israel

\section{Strikman}

Department of Physics, Pennsylvania State University, University Park, PA 16802, USA

ABstract: We discuss nuclear broadening of the intrinsic transverse momentum of partons in the initial state, and its influence on the $p_{t}$ distribution of prompt photons. Comparing to WA98 data from $\mathrm{Pb}+\mathrm{Pb}$ collisions at $\sqrt{s}=17.4 A \mathrm{GeV}$ we find evidence for the presence of nuclear broadening at high $p_{t}$ in this hard process. Below $p_{t} \sim 2.7 \mathrm{GeV}$ the photon distribution is due to small momentum transfer processes. At RHIC energy, $\sqrt{s}=200 A \mathrm{GeV}$, the effect of intrinsic transverse momentum on the spectrum of prompt photons is less prominent. Below $p_{t}=2-3 \mathrm{GeV}$ the contribution from large momentum transfers flattens out, and we expect that region to be dominated by soft contributions.

\section{Introduction}

The WA98 collaboration recently reported data on direct photon production in lead on lead collisions at CERN SPS energies $(\sqrt{s}=17.4 A \mathrm{GeV})$ at $p_{t} \sim 1.5-4 \mathrm{GeV}$ [i].]. They made the interesting observation that in central $\mathrm{Pb}+\mathrm{Pb}$ collisions the multiplicity of direct

\footnotetext{
${ }^{*}$ Speaker.

${ }^{\dagger}$ LG wants to thank the Josef-Buchmann foundation, the "Vereinigung von Freunden und Förderern der Johann Wolfgang Goethe-Universität" and the Organizers of HEP2001 for financial support.
} 
photons per nucleon-nucleon collision in that range of $p_{t}$ is enhanced relative to protonproton collisions at similar energy.

For $p_{t} \gtrsim 2.7 \mathrm{GeV}$ the enhancement may be explained by the nuclear broadening of the intrinsic transverse momenta of the partons in the initial state [2i]. At smaller $p_{t}$, the prompt photon cross section is dominated by small, presumably non-perturbative momentum transfers. Intrinsic transverse momentum plays a less important role at higher energies.

\section{Proton-Proton Collisions}

We consider the contributions from Compton-like scattering $(g+\stackrel{(-)}{q} \rightarrow \gamma+\stackrel{(-)}{q})$, annihilation $(q+\bar{q} \rightarrow \gamma+g)$, plus collinear bremsstrahlung off a (anti-)quark produced at midrapidity. Assuming the applicability of the QCD factorization theorem, the corresponding expressions for the Compton and annihilation subprocesses in $p+p \rightarrow \gamma+X$ are [iji]

$E \frac{\mathrm{d} \sigma_{\gamma}}{\mathrm{d}^{3} p}=\sum \int \mathrm{d} x_{a} \mathrm{~d} x_{b} \mathrm{~d}^{2} k_{t a} \mathrm{~d}^{2} k_{t b} f\left(k_{t a}\right) f\left(k_{t b}\right) G_{a / A}\left(x_{a}, Q^{2}\right) G_{b / B}\left(x_{b}, Q^{2}\right) \frac{\hat{s}}{\pi} \frac{\mathrm{d} \sigma}{\mathrm{d} \hat{t}} \delta(\hat{s}+\hat{t}+\hat{u}) \Theta_{0}$.

$\hat{s}, \hat{t}, \hat{u}$ denote the Mandelstam variables for the $a+b \rightarrow \gamma+c$ elementary process. The sum extends over all possible partons in the initial state, i.e. gluons and $u, d, s$ (anti-)quarks, and over any final state interactions. $\mathrm{d} \sigma / \mathrm{d} \hat{t}$ denotes the elementary hard-scattering cross section for the corresponding process, averaged over all possible spin and color orientations in the initial state, and summed over those in the final state. Explicit expressions can be found e.g. in $[\bar{\beta} \bar{\beta}]$.

The functions $f\left(k_{t}\right)$ parametrize the transverse momentum of the partons in the initial state. For simplicity, we assume a Gaussian distribution, which contains one tunable parameter, namely the average intrinsic transverse momentum of the partons in the initial state, $\left\langle k_{t}^{2}\right\rangle$. We shall present results for various values of $\left\langle k_{t}^{2}\right\rangle$ below. The limit $\left\langle k_{t}^{2}\right\rangle \rightarrow 0$ recovers the usual collinear factorization.

For the factorization scale we assume $Q^{2}=\left(2 p_{t}\right)^{2}$, and as we restrict ourselves to the leading logarithmic approximation, we assume that the renormalization scale is the same. We employ the one-loop expression for the running coupling constant $\alpha_{s}\left(Q^{2}\right)$ for $N_{f}=4$, and $\Lambda_{\mathrm{QCD}}=236 \mathrm{MeV}$, as appropriate for CTEQ4L. Collinear divergencies arising from partonic processes with large intrinsic transverse momentum are cut off by

$$
\Theta_{0}=\Theta_{1} \Theta_{2} \Theta\left(Q_{c}^{2}-1 \mathrm{GeV}^{2}\right) \text { with } Q_{c}^{2}=\frac{2 \hat{s} \hat{t} \hat{u}}{\hat{s}^{2}+\hat{t}^{2}+\hat{u}^{2}} .
$$

In this way, only subprocesses with momentum transfer $\geq 1 \mathrm{GeV}$ are taken into account, for which one can hope that factorization is a reasonable assumption. The functions $\Theta_{1}$ and $\Theta_{2}$ ensure that parton $a$ is moving to the right, while parton $b$ is moving to the left.

Collinear bremsstrahlung is computed as follows. First, the cross-section for (anti)quark production at $y_{c m}=0$ with transverse momentum $p_{t c}$ is computed in the standard fashion, as described above. This is then convoluted with the QED collinear fragmentation 
function $z_{c} D_{\gamma / q}\left(z_{c}\right)=\alpha\left[1+\left(1-z_{c}\right)^{2}\right] \log \left(p_{t}^{2} / \Lambda_{\mathrm{QCD}}^{2}\right) / 2 \pi$ into $\gamma+X$, where $z_{c}=p_{t} / p_{t c}$

$$
\begin{aligned}
E \frac{\mathrm{d} \sigma_{\gamma}}{\mathrm{d}^{3} p}= & \sum \int \mathrm{d} x_{a} \mathrm{~d} x_{b} \mathrm{~d} z_{c} \mathrm{~d}^{2} k_{t a} \mathrm{~d}^{2} k_{t b} \\
& f\left(k_{t a}\right) f\left(k_{t b}\right) G_{a / A}\left(x_{a}, Q^{2}\right) G_{b / B}\left(x_{b}, Q^{2}\right) D_{\gamma / c}\left(z_{c}\right) \frac{\hat{s}}{\pi z_{c}^{2}} \frac{\mathrm{d} \sigma}{\mathrm{d} \hat{t}} \delta(\hat{s}+\hat{t}+\hat{u}) \Theta_{0} .
\end{aligned}
$$

Here, we employ $Q^{2}=\left(2 p_{t c}\right)^{2}$ for the factorization and renormalization scale of the hard process. For the bremsstrahlung contribution we do not sum explicitly over all hard subprocesses leading to the production of a (anti-)quark at midrapidity but rather employ the method of effective structure function,

$$
G_{a / A}\left(x_{a}, Q^{2}\right) G_{b / B}\left(x_{b}, Q^{2}\right)=\left(g\left(x_{b}, Q^{2}\right)+\frac{4}{9} \sum_{f} q_{f}\left(x_{b}, Q^{2}\right)\right) \sum_{f} e_{f}^{2} q_{f}\left(x_{a}, Q^{2}\right),
$$

where $e_{f}$ is the fractional electric charge of the splitting (anti-)quark. Further, $\mathrm{d} \sigma / \mathrm{d} \hat{t}=$ $\pi\left(\alpha_{s} / \hat{s}\right)^{2}\left(\hat{s}^{2}+\hat{u}^{2}\right) / \hat{t}^{2}$. The $\Theta$-function is defined as in $\left(\overline{2}_{2}^{2} \cdot 2_{1}^{i}\right)$. Note that in these expressions $\hat{s}, \hat{t}, \hat{u}$ refer to the partonic subprocess, "before" splitting of the final-state (anti-)quark into $\gamma+X$.

It is common practice to multiply the r.h.s. of $(\overline{2} .11)$ and $(2.2 .3)$ by a $K$ factor in order to account for NLO contributions. We use in this work $K=$ 2. Our major interest is in the relative yields of prompt photons in $p+p$ and in $A+A$ collisions, and in that sense the $K$-factor plays no essential role.

In Fig. $]_{1}^{1}$ we compare our results for $p+p$ at $\sqrt{s}=19.4 \mathrm{GeV}$, for various intrinsic $\left\langle k_{t}^{2}\right\rangle$, to E704 data [i $[\overline{4}]$. Clearly, the calculation assuming pure DGLAP evolution, i.e. $\left\langle k_{t}^{2}\right\rangle=0$, falls short by almost an order of magnitude. The best fit is obtained using $\left\langle k_{t}^{2}\right\rangle=1.3-1.5 \mathrm{GeV}^{2}$.

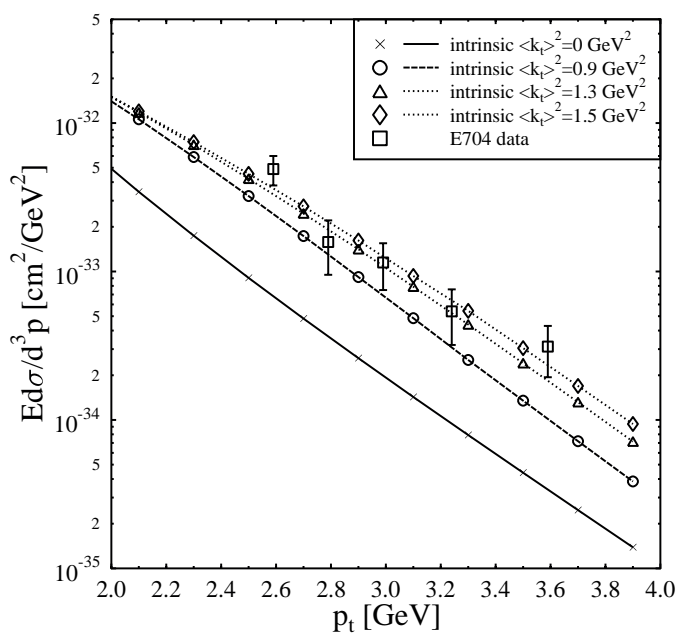

At first sight this appears somewhat larger than the "standard" $\left\langle k_{t}^{2}\right\rangle=0.9 \mathrm{GeV}^{2}$ from [i3]. However, recall that we employ a larger $Q^{2}=4 p_{t}^{2}$ scale than [i3]

Figure 1: The differential direct photon cross section vs. $p_{t}$ (at center-of-mass rapidity) for proton-proton who used $Q^{2}=p_{t}^{2} / 2$. In accord with collsions at $\sqrt{s}=19.4 \mathrm{GeV}$.

the picture that the intrinsic transverse momentum is due mainly to gluon radiation in the initial state, a larger $Q^{2}$ scale therefore has to correspond to larger $\left\langle k_{t}^{2}\right\rangle$. This general expectation is confirmed by our results. (The value for $\left\langle k_{t}^{2}\right\rangle$ therefore only acquires physical meaning together with a definition of $Q^{2}$.) A value of $\left\langle k_{t}^{2}\right\rangle \simeq 1.3-1.5 \mathrm{GeV}^{2}$ is required to fit the data at that energy. 


\section{Nucleus-Nucleus Collisions}

To extend our calculations to nucleus-nucleus collisions we have to substitute the parton distribution functions $G_{N}(x, Q)$ in eq. (2.2. $\left.\overline{2}^{\prime}\right)$ by $G_{A}\left(x, Q, \mathbf{b}^{\prime}\right)$ for the projectile and $G_{B}\left(x, Q, \mathbf{b}-\mathbf{b}^{\prime}\right)$ for the target nucleus. Here, $G_{A}\left(x, Q, \mathbf{b}^{\prime}\right)=A \cdot T_{A}\left(\mathbf{b}^{\prime}\right) G_{N}(x, Q)$ and $G_{B}\left(x, Q, \mathbf{b}-\mathbf{b}^{\prime}\right)=B \cdot T_{B}\left(\mathbf{b}-\mathbf{b}^{\prime}\right) G_{N}(x, Q)$, with $\mathbf{b}$ the nucleus-nucleus impact parameter, and $\mathbf{b}^{\prime}$ the distance from the center of the projectile. Note that $\mathbf{b}$ and $\mathbf{b}^{\prime}$ are two-dimensional vectors.

The nuclear thickness functions $T_{A, B}\left(\mathbf{b}^{\prime}\right)$ are defined as

$$
T_{A, B}\left(\mathbf{b}^{\prime}\right)=\int \mathrm{d} z \rho_{A, B}\left(\mathbf{b}^{\prime}, z\right) \text { with } \int \mathrm{d}^{2} \mathbf{b}^{\prime} \mathrm{d} z \rho\left(\mathbf{b}^{\prime}, z\right)=1 .
$$

$A(B)$ are the number of nucleons in the projectile (target). We account for the dependence on the nucleus nucleus impact parameter $b$ as due to the geometry of the collision only. More details are given in ref. [2]2]

Fig. ${ }_{2}^{2}$ dic depicts the differential multiplicity of direct photons vs. transverse momentum in the $10 \%$ most central $\mathrm{Pb}+\mathrm{Pb}$ reactions at $\sqrt{s}=17.4 A \mathrm{GeV}$. Again, as was the case for $p+p$ collisions at similar energy, the calculation assuming pure DGLAP evolution underestimates the data strongly. However, the "standard" intrinsic transverse parton momentum of $\left\langle k_{t}^{2}\right\rangle \simeq 1.3 \mathrm{GeV}^{2}$ extracted already on the $p+p$ level improves the agreement a lot. Within the experimental error bars, the WA98 data leaves room for up to $\Delta k_{t}^{2} \sim 1 \mathrm{GeV}^{2}$ additional broadening from nuclear effects.

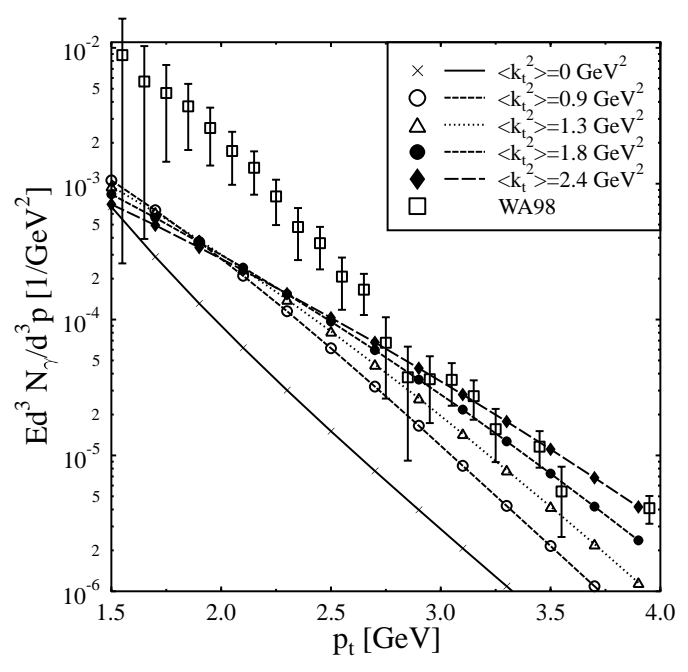

However, we can also conclude that transverse momentum broadening much beyond $\Delta k_{t}^{2} \simeq 1 \mathrm{GeV}^{2}$ is not seen in the WA98 data.

Another interesting result is that the calculated photon spectrum below $p_{t} \lesssim 2.5 \mathrm{GeV}$ depends only weakly on the amount of intrinsic transverse momentum. That is because of the cut-off on the momentum transfer, eq. (2.2). The excess photons seen in the data, as compared to our calculation, can be attributed to small-momentum transfer scatterings, and so are presumably dominated by final-state interactions.

In Fig. $\overline{3}_{-1}$ we show prompt photon spectra at RHIC energy, for the $10 \%$ most central $\mathrm{Au}+\mathrm{Au}$ events at $\sqrt{s}=200 \mathrm{~A} \mathrm{GeV}$. One observes that the effect of intrinsic transverse 
momentum in the initial state is much less prominent than at lower energy, in agreement with the results of [5] for pion production. The reason for this behavior is that the photon spectrum becomes "harder" at higher energy, i.e. it decreases less steeply with $p_{t}$. Nevertheless, at $p_{t} \sim 3-4 \mathrm{GeV}$ the intrinsic parton transverse momentum can increase the prompt photon multiplicity by up to a factor of 3 . That would be the most promising kinematical domain for an experimental study of the nuclear broadening of the intrinsic transverse momentum distributions. The region of smaller $p_{t}$ might be dominated by soft contributions, as indicated by the flattening of the spectra in Fig. ${ }_{3}^{3} \cdot$. On the other hand, at larger $p_{t}$ intrinsic transverse momentum becomes less effective.

\section{Conclusions}

- The calculated photon production agrees with the proton-proton data.

- With broadening, WA98 data at $p_{t}>2.5 \mathrm{GeV}$ can be explained within pQCD.

- Photon production at smaller $p_{t}$ is dominated by nonperturbative processes with small momentum transfer $\left(Q<1 \mathrm{GeV}^{2}\right)$.

- The intrinsic $k_{t}$ changes the calculated photon yield by an order of magnitude.

The required broadening is in agreement with proton-nucleus data.

- At BNL-RHIC the change due to the broadening is smaller than at SPS.

-The best place to search for this effect at the BNL-RHIC is $3<p_{t}<4$ $\mathrm{GeV}$.

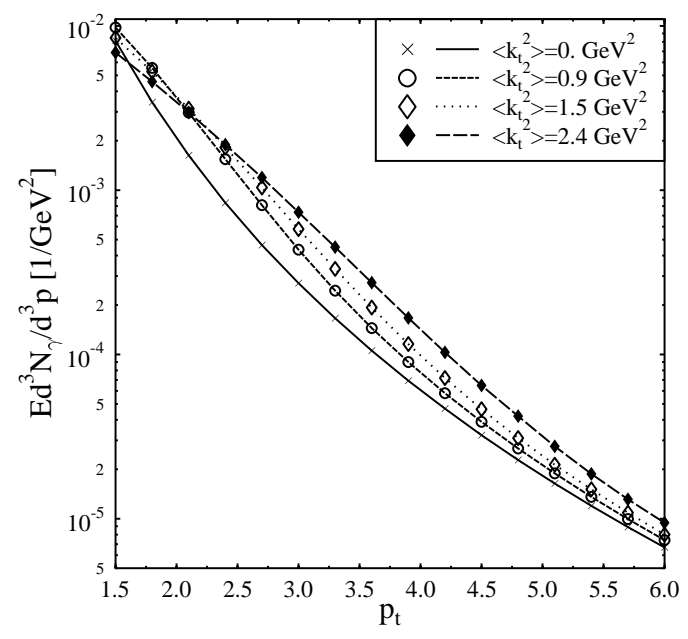

Figure 3: The transverse momentum distribution of prompt photons at center-of-mass rapidity in the $10 \%$ most central $\mathrm{Au}+\mathrm{Au}$ reactions at $\sqrt{s}=200 \mathrm{~A} \mathrm{GeV}$ for various intrinsic $\left\langle k_{t}^{2}\right\rangle$.

\section{References}

[1] M. M. Aggarwal et al. [WA98 Collaboration], PPhys. Rev. Lett. 85 $(200 \overline{0}) \overline{3} 5 \overline{5} \overline{1}$

[2] A. Dumitru, L. Frankfurt, L. Gerland, H. Stöcker, M. Strikman, 'Pिhys. Rev. $\mathbf{C} \mathbf{6} \overline{4}(\overline{2} \overline{0} \overline{1} \overline{1}) !$ -

[3] J. F. Owens, Rev. Mod. Phys. $\mathbf{5 9} 9(1987) 465$

[4] D. L. Adams et al. [E704 Collaboration], Phys. Lett. $\mathbf{B}-\mathbf{3} \mathbf{4} 5 \overline{5} 1995) 569_{1}^{\prime}$

[5] X. N. Wang, 'Phys. Rev. Lett. 81 (1998) 2655; M. Gyulassy and P. Levai, 'Ṕ in 